\title{
Mimèsis et comédie: déterminations de l'ancienne querelle entre philosophie et théâtre
}

\author{
María J. Ortega Máñez
}

\section{(2) OpenEdition}

\section{Journals}

Édition électronique

URL : https://journals.openedition.org/edl/3146

DOI : $10.4000 /$ edl. 3146

ISSN : 2296-5084

Éditeur

Université de Lausanne

\section{Édition imprimée}

Date de publication : 24 septembre 2020

Pagination : 45-58

ISBN : 978-2-940331-74-1

ISSN : 0014-2026

\section{Référence électronique}

María J. Ortega Máñez, « Mimèsis et comédie: déterminations de l'ancienne querelle entre philosophie et théâtre », Études de lettres [En ligne], 313 | 2020, mis en ligne le 24 septembre 2021, consulté le 25 septembre 2021. URL : http://journals.openedition.org/edl/3146; DOI : https://doi.org/10.4000/edl. 3146 


\section{MIMĖSIS ET COMÉDIE: \\ DÉTERMINATIONS DE L'ANCIENNE QUERELLE ENTRE PHILOSOPHIE ET THÉÂTRE}

L'ancienne querelle entre philosophie et théâtre est réévaluée ici à la lumière d'une hypothèse de Denis Guénoun, selon laquelle "la comédie est le devenir-théâtre du théâtre». Quatre constats mis en évidence permettent de déterminer les pratiques examinées en tant que mimèsis (pour la philosophie) et comédie (pour le théâtre). Un corollaire portant sur le théâtre classique espagnol fournit une preuve supplémentaire à cette argumentation, qui a la forme logique finale d'un syllogisme ${ }^{1}$.

Si les termes "philosophie» et "théâtre» formant l'intitulé de cette rencontre comportaient des majuscules, comme il est d'usage en langue allemande, on serait tenté d'y voir deux noms propres qui, liés par la conjonction, et suivis du sous-titre "Scènes de jeu " personnifiant un rapport, joueraient quelque chose: un «auto sacramental» peut-être ou une comédie de boulevard.

Sans trop céder à une certaine tendance de la pensée à la figuration théâtrale - versant de la philosophie relevé, entre autres, par Deleuze sous la désignation de "personnages conceptuels» ${ }^{2}-$, on peut aussi entendre "philosophie» et "théâtre" comme des pratiques auxquelles les philosophes d'une part, et les poètes et praticiens de la scène de l'autre se sont voués depuis bien longtemps. Le résultat de ce "faire», cristallisé dans des œuvres, peut donc être étudié dans une perspective historique. Deux modalités majeures d'interaction se révèlent alors: la querelle

I. Je remercie Vincent Verselle pour sa relecture.

2. G. Deleuze, F. Guattari, Qu'est-ce que la philosophie?, p. 60-81. 
(dans les grandes lignes, lorsque les poètes attaquent les philosophes et les philosophes critiquent le théâtre) et l'association, voire la synthèse ${ }^{3}$.

On se concentrera ici sur la première. Nous aborderons la querelle entre philosophie et théâtre - qui constitue dans son contexte grec d'origine le sujet de notre thèse de doctorat - à la lumière de nos recherches récentes portant sur le comique. Sous le signe d'une hypothèse théorique empruntée à Denis Guénoun, nous tenterons une vérification: tout en revenant sur quelques constats issus de notre analyse de l'ancienne querelle entre théâtre et philosophie, il s'agira de préciser ici les termes de la relation: qu'est-ce qui, dans la philosophie et dans le théâtre, intervient dans cet ancien différend?

\section{Hypothèse: la comédie est le proprement théatral}

Nous convoquons tout d'abord une idée de Denis Guénoun élevée à la catégorie d'hypothèse. Quoiqu'esquissée ici et là dans ses écrits et enseignements ${ }^{4}$, elle est formulée dans un texte paru récemment, court et comme le note Michael Groneberg "radical ${ }^{5}-$ au sens étymologique, osons-nous l'entendre: se situant à la racine du théâtre dans son rapport à la poésie. Le texte en question ${ }^{6}$ expose cinq propositions fondamentales. La première, en amorce, établit que l'écriture théâtrale est d'essence poétique. Suivent quatre acceptions ou extensions de cette formule qui découle à son tour d'une évidence: les plus grands textes de théâtre ont été des poèmes. La comédie paraît prosaïque, mais ne l'est pas. Les premiers comiques, en ordre chronologique et hiérarchique, sont des poètes. Le poème est le dire initial; en théâtre, et peut-on ajouter en

3. Plusieurs sous-types en rendent compte. Succinctement: a) des philosophes qui ont pensé le théâtre pour le décrire ou en prescrire de meilleures formules de production: Aristote, Diderot, Hegel; b) des philosophes qui ont écrit des pièces de théâtre: Voltaire, Sartre, Camus, Badiou; c) des dramaturges mettant en scène des thèses philosophiques: Calderón de la Barca (La vida es sueño, El gran teatro del mundo), s'inspirant de lui, Hugo von Hofmannsthal (Jedermann, Das Salzburger Große Welttheater), le Bertolt Brecht des Lehrstücke ou Jean-Claude Carrière (La controverse de Valladolid).

4. Cf. D. Guénoun, Le théâtre est-il nécessaire?, p. 148 ss.; séminaires tenus à ParisSorbonne (Paris IV) entre 2007 et 2011.

5. M. Groneberg, «Introduction", p. 13.

6. D. Guénoun, "Théâtre et poésie», p. 170-179. 
philosophie - que l'on pense à Parménide et à certains Présocratiques: s'ils écrivent, c'est en vers. Celui-ci est le point de départ de trois extensions du poème: vers la scène, vers la comédie et vers la prose.

Le poème est donc porté vers la scène, porté à se dire à voix haute, à se montrer, à se donner à voir et à entendre en même temps. Pourquoi le théâtre veut-il le poème? demande Guénoun. À cette question, il répond:

Le thêâtre ne veut pas le poème, parce que le théâtre ne veut rien. Le théâtre n'est pas une pulsion, une volonté, une personne. À strictement parler, le théâtre n'est rien. Au moins par lui-même. Le théâtre n’a pas d'essence. Au moins en propre. L'essence du théâtre, c'est le poème. Le théâtre n'est rien d'autre que le mouvement qui porte le poème à s'exposer ${ }^{7}$.

Ce mouvement est caractérisé chez lui à l'aide de la figure de l'hypotypose, sur laquelle est centrée la seconde proposition: «le théâtre relève l'hypotypose» qui habite le poème. Il actualise littéralement ce désir poétique de donner à voir, d'où naît la tragédie. Vient alors la deuxième extension: dans ce mouvement vers la scène que le théâtre noue avec la tragédie, pourquoi ensuite la comédie? L'auteur soutient que la comédie, qui naît comme commentaire parodique de la tragédie, désignerait l'autonomisation de ce processus d'exposition, de devenir-visible.

Le théâtre qui, au sein de la tragédie n'existait pas en propre, n'avait pas d'essence, s'affirme dans son être comme comédie. Proposition 3: La comédie est le devenir-théâtre du théâtre ${ }^{8}$.

Pourquoi? Guénoun en donne les raisons.

2. Vérification: la querelle entre philosophie et théatre en Grèce classique. Déterminations

Nous proposons ici un chemin d'argumentation alternatif pour démontrer cette thèse. Il a comme point de départ la querelle entre philosophie

7. Ibid., p. 172.

8. Ibid., p. 174. 
et théâtre en Grèce classique, qui peut se résumer comme suit ${ }^{9}$ : clôturant son examen de la mimèsis poétique dans La République de Platon, Socrate affirme que «la querelle est ancienne entre la philosophie et la création poétique» (X, 607b). L'ancienneté de la querelle et son existence sont ainsi postulées d'un même coup. Or, une enquête auprès des sources révèle que le seul précédent de ce différend est constitué par les Nuées d'Aristophane (423 av. J.-C.). En quoi cette comédie constitue-t-elle une attaque contre la philosophie? Notre recherche a soulevé deux enjeux majeurs qui caractérisent la spécificité du geste d'Aristophane au sein de cette querelle.

D'abord la création du personnage comique du philosophe, en l'occurrence Socrate. Premier d'une longue tradition, Socrate est présenté par Aristophane sous les traits d'un sophiste et d'un philosophe de la nature. Retiré dans son pensoir, il mesure la distance dans le saut d'une puce et enseigne des techniques rhétoriques capables de faire remporter n'importe quelle cause dans les tribunaux. Enjeu politique, en effet: un aperçu historique des comédies portant sur ce sujet, et des décrets promulgués à la même époque dans le but de régulariser la situation politique déclenchée par celles-ci, permet de mesurer l'ampleur d'un phénomène social aux lourdes conséquences. De surcroît, Aristophane est cité explicitement dans l'Apologie de Socrate pour avoir contribué à forger la mauvaise réputation qui mena à sa mise en accusation et à son exécution.

Ensuite, à titre d'enjeu philosophique, nous avons mis en avant l'invention aristophanienne du chœur des Nuées qui instituent la mimèsis comme fondement de la création poétique. Par leur capacité à prendre toute figure dans le but de se moquer des excès de la société, les Nuées, que Socrate vénère et dont Aristophane (en tant que coryphée) fait partie, sont les déesses de l'imitation poético-philosophique. Elles posent donc la question de la création poétique en terme d'imitation.

La riposte vient de Platon. Les signes de son hostilité envers Aristophane se trouvent dans l'Apologie de Socrate et le Banquet. Mais le problème de plus grande envergure, concernant et le théâtre et le difficile rapport du philosophe avec la cité, est traité dans La République qui tente de répondre à la question de ce qu'est la justice à travers la fondation en

9. Pour une synthèse, cf. notre position de thèse: Academia.edu> María J. Ortega Máñez> PhD. 
discours d'une cité parfaite, où cette qualité serait à l'ordre. C'est dans ce dialogue que le problème du rôle du philosophe dans la cité, soulevé par les Nuées d'Aristophane et rendu vital par la mort de Socrate, est réglé. C'est ici que se trouvent la célèbre thèse du philosophe-roi et le - discutable - bannissement de la poésie mimétique de la kallipolis, suite à un examen et à une critique en deux temps de la mimèsis. De cet état de choses, il convient de soulever ici quelques constats.

\subsection{Concernant l'origine du terme mimèsis.}

Mimèsis dérive du substantif mîmos, qui aurait désigné au $\mathrm{IV}^{\mathrm{e}}$ siècle av. J.-C. autant le genre des «mimes" que l'interprète de ces pièces, une "espèce d'acteur qui récite, chante et danse ${ }^{10}$. Ce genre dramatique est né dans le contexte dorien ${ }^{11}$, plus probablement en Sicile. Aristote cite précisément les mimes dans sa Poétique ${ }^{12}$, à titre de genre poétique. Ils consistaient dans "des scènes de la vie quotidienne et non, contrairement à un malentendu récurrent, des mimes muets au sens moderne» ${ }^{13}$.

D'après ce que l'on sait des mimes - au premier sens du mot -, il devait s'agir autant de récits avec des parties jouées par un acteur que de performances dramatiques exécutées par deux voire plusieurs acteurs. Considérés comme des artistes scéniques, ces mimes - selon la seconde acception - ont, avec les acteurs de tragédie et de comédie, quelques points communs: ils jouent dans un décor scénique, se barbouillent le visage ou portent parfois le masque, mais pas de brodequin ni de cothurne ${ }^{14}$. Selon Paulette Ghiron-Bistagne, "la différence entre les

Io. P. Chantraine, Dictionnaire étymologique de la langue grecque, p. 703.

II. Tout comme, selon Aristote, la tragédie et la comédie: "Les Doriens revendiquent pour eux la tragédie et la comédie [...]. Ils invoquent comme preuve les noms: en effet ils disent qu'ils appellent les faubourgs kômai - tandis que les Athéniens les appellent "dèmes» - et que le nom de "comédien» vient non de kômazein, mais de ce que, interdits de séjour dans la ville, ils allaient de kômè en kômè» (Poétique 3, 1448a3038).

I2. "L'art qui fait usage seulement du langage en prose, ou des vers, et qui, dans ce dernier cas, peut combiner entre eux différents mètres ou n'en utiliser qu'un seul» et qui «n’a pas reçu de nom jusqu’à présent. Car nous n'avons pas de terme commun pour désigner à la fois les mimes de Sophron et de Xénarque et les dialogues socratiques» (Poétique 1, 1447a28 - b10-11).

I3. S. Halliwell, The Aesthetics of Mimesis, p. 17.

I4. Cf. É. Littré, Dictionnaire de la langue française, IV, art. «mime», p. 3899. 
vrais acteurs et ces artistes pseudo-dramatiques tient aussi à des raisons agonistiques " ${ }^{15}$. Les mimes se produisaient devant des particuliers, probablement lors de banquets et d'autres occasions festives de la haute société, et leurs sujets étaient tirés du champ de ce que les interprètes anglo-saxons appellent la "low-life», soit "le comportement des gens qui n’appartiennent pas aux couches riches de la société et n'affichant donc pas de vertus aristocratiques " ${ }^{16}$. "Un lâche au milieu d'une bataille» ou «un homme en train de voler des vaches» en sont des exemples.

La proximité de la réalité historique des mimes avec la définition de la comédie donnée par Aristote dans La Poétique en tant que «représentation d'hommes bas" (mimêsis phauloterôn, 1449a32) est remarquable.

\subsection{Mimèsis comme ressource comique par excellence}

Faire comme quelqu'un d'autre, voire se faire passer pour quelqu'un d'autre au moyen de l'apparence et le jeu; cette ligne de signification du terme mimèsis et de ses dérivés s'affirmant, la mimesis devient une ressource privilégiée de la comédie. On en trouve plusieurs exemples chez Aristophane.

Dans L'Assemblée des femmes, les Athéniennes décident de se rendre à la place de leurs maris à l'Assemblée afin de se saisir du gouvernement de la cité et de voter des lois enfin justes. La première disposition à prendre est précisément d'adopter l'apparence des hommes pour accéder aux lieux du pouvoir. Praxagora, la présidente des conjurées, dirige ainsi l'opération de travestissement, qui pivote autour du verbe mimeisthai:

Allons, relevez vos petites tuniques, chaussez au plus vite vos laconiennes, comme vous le voyez faire à votre mari chaque fois qu'il doit se rendre à l'Assemblée ou sortir. Puis, tout cela une fois en bon ordre, attachez vos barbes; et quand vous les aurez soigneusement ajustées, prenez encore les manteaux d'hommes que vous avez dérobés et rejetez-les sur l'épaule; ensuite, appuyées sur vos bâtons, marchez en chantant quelque refrain de vieux, imitant (mimoúmenai) la manière des campagnards ${ }^{17}$.

15. P. Ghiron-Bistagne, Recherches sur les acteurs en Grèce antique, p. 3.

16. G. Sörbom, Mimesis and Art, p. 23. Nous traduisons.

17. Aristophane, L'Assemblée des femmes, vv. 268-278, p. 26-27. 
Encore un exemple, tiré des Grenouilles. En vue de son imminente descente aux Enfers, Dionysos apparaît déguisé en Héraclès pour demander conseil à celui qui a réussi à en revenir vivant:

DIONYSOS. Mais les raisons qui m'amènent avec cet accoutrement imité (mímêsin) du tien, c'est pour que tu m'indiques, en cas de besoin, les hôtes qui t'ont servi, quand tu allas chercher Cerbère.

HÉRACLÈS. Pas moyen de chasser ce rire, quand je vois une peau de lion par-dessus une crocote! Que veut dire cela? Que vient faire un cothurne avec une massue? ${ }^{18}$

Le déguisement de Dionysos est tellement grotesque qu'Héraclès a du mal à s'arrêter de rire. Il est notamment rendu ridicule par la superposition des attributs du héros mythique avec ceux de l'acteur dans un ensemble bigarré. Ceci montre que ce genre d'imitation, bien qu'ayant un modèle reconnaissable, ne vise pas sérieusement à supplanter l'original: l'effet comique réside justement là. Comme le remarque Michael Groneberg, le rapport double entre objet-modèle et objet-copie est plus clair dans le cas de la comédie:

Le Socrate suspendu entre le ciel et la terre dans les Nuées est plus clairement différent du Socrate-modèle que le sont les Socrates des dialogues de Platon. On peut estimer plus haut le Socrate idéalisé de Platon que celui, plus réaliste, de Xénophon, comme le fait Sören Kierkegaard ${ }^{19}$.

Si la référence historique ou mythologique de ces exemples gêne la clarté, pensons à la scène du miroir de Duck Soup, mémorablement jouée par Groucho Marx: le comique est précisément déclenché par l'écart entre le modèle et la copie.

En définitive, lorsque le terme mimèsis est utilisé à l'intérieur d'une fable comique, il dénote souvent un processus de déguisement en vue d'un dessein majeur - d'habitude une ruse adressée à un autre personnage. Ce n'est pas la perfection du travestissement qui est donc envisagée. L'explicitation du modèle aux yeux des spectateurs a justement pour fonction de révéler ce processus, de montrer le travestissement en

I8. Aristophane, Grenouilles, vv. 109, 45-47, respectivement; p. 90, 87.

I9. M. Groneberg, «La mimesis», p. 160. 
tant que travestissement ${ }^{20}$. Le recours au déguisement, plus ou moins explicite, sera monnaie courante dans la comédie postérieure.

\subsection{Mimèsis comme jeu chez Platon}

Dans l'évolution sémantique du mot mimèsis, Platon aura un rôle décisif, car non seulement il élargit considérablement son registre de sens, mais il crée aussi le concept et lie inéluctablement sa destinée à celle de la philosophie.

Il est intéressant d'observer qu'à l'occasion de la dévaluation ontologique de la mimèsis au livre $\mathrm{X}$ de La République, celle-ci était caractérisée en tant que «jeu»:

L'imitation est un jeu puéril, pas une chose sérieuse (einai paidian tina kai ou spoudên tên mimêsin) ${ }^{21}$.

Platon inscrit la mimèsis artistique dans le champ de l'amusement, comme il l'affirmera nettement plus tard:

Toutes les imitations que produisent peinture et musique n'ont d'autre but que notre plaisir et il est juste de les rassembler sous un seul nom: celui de divertissement (paignion) ${ }^{22}$.

Curieusement, cette même idée de "jeu» est utilisée par Socrate, à un instant crucial de La République, pour définir métathéâtralement son propre exercice de la philosophie:

- J'ai l'impression que c'est moi-même, pour l'instant, qui suis ridicule (geloion).

- En quoi? dit-il.

20. C'est Ricœur, à notre connaissance, qui a insisté le plus sur le caractère actif de la mimesis qui lui confère son association à poiêsis dans les ultérieures conceptualisations philosophiques mais qui est déjà inhérent au suffixe -is. Quoique l'élucidant par rapport à son élaboration aristotélicienne, sa précision nous semble bien s'accorder avec cette acception originaire que nous commentons: "la même marque doit être conservée dans la traduction de mimèsis: qu'on dise imitation ou représentation (avec les derniers traducteurs français), ce qu'il faut entendre, c'est l'activité mimétique, le processus actif d'imiter ou de représenter. Il faut donc entendre imitation ou représentation dans son sens dynamique de mise en représentation, de transposition dans des œuvres représentatives» (P. Ricœur, Temps et récit, I, p. 69).

2I. Platon, République X, 602b.

22. Platon, Politique, 288c. 
- J'avais oublié, dis-je, que nous étions en train de jouer (epaizomen), et j'ai trop haussé le ton. En effet, en parlant j'ai regardé du côté de la philosophie, et en la voyant indignement couverte de boue, il me semble que je me suis emporté, et que j'ai dit ce que j'ai dit en me mettant en colère contre ceux qui en sont la cause, avec trop de sérieux (spoudaioteron $)^{23}$.

Il ne faut pas oublier que l'ironie est trait distinctif et méthode chez Socrate, ce qui signifie que parfois il faut interpréter ce qu'il dit comme son contraire. Le ridicule, comme l'ignorance avouée, est chez Platon marque de sagesse. Ce qui nous importe de souligner ici, c'est l'assimilation du théâtre - ou de la mimèsis poétique - et de la philosophie, en vertu d'un certain caractère ludique commun.

En bref, le rapport de la mimèsis avec le comique est constitutif du mot - pratique et théorie. C'est pourquoi on le retrouve tantôt à l'origine - les «mimes» -, tantôt au sein de la comédie - Aristophane. La mimèsis entendue comme jeu chez Platon garde quelque chose du comique originaire, mais dilué dans le champ sémantique plus général du ludique qui peut même relier, quoique moyennant l'ironie, ce que Platon disjoint: la poésie et la philosophie. Cette connotation sera perdue dans le passage à Aristote, où mimèsis n'a plus de connotation ontologique négative et sert à définir autant la tragédie que la comédie et, par conséquent, à constituer le concept pivot qui dit la création théâtrale.

\section{4. Théâtre déterminé comme comédie}

Soulignons enfin que ce qu'on a désigné jusque-là sous le nom général de "théâtre" s'avère concrètement en tant que théâtre comique. Autrement dit, la querelle ancienne entre philosophie et théâtre est dans les faits la querelle entre la philosophie platonicienne et un aspect du théâtre comique d'Aristophane. Un renouveau de cette querelle aura lieu au XVIII ${ }^{e}$ siècle en France. Un certain nombre d'auteurs comiques Palissot à leur tête, avec sa comédie Les Philosophes (1760) - raillent sur les planches les Encyclopédistes - surtout Diderot, Voltaire, Rousseau ${ }^{24}$ - , tandis qu'une nouvelle condamnation des spectacles théâtraux voit le

23. Platon, République (VII, 536b-c), p. 394-395.

24. On peut lire le détail de cette querelle dans l'introduction et la chronologie d'Olivier Ferret (Palissot, La comédie des Philosophes et autres textes). 
jour sous la plume de Rousseau, qui les juge nuisibles dans le cadre de sa république natale ${ }^{25}$. C'est le théâtre comique, dans les deux cas, qui met en crise la philosophie.

\section{Corollaire: le théatre classique espagnol}

En guise de corollaire à ce raisonnement, survolons la vaste et variée production du théâtre espagnol baroque. Il est frappant d'observer que l'ensemble de ce théâtre, sauf le religieux, est "comédie», ou du moins ainsi s'énonce-t-il: comedia. On trouve très rarement tragedia dans les autodénominations. Par exemple: le manuscrit d'El médico de su honra (1637), une des pièces les plus noires de Calderón de la Barca, présente au-dessus de son titre l'appellatif "comedia famosa", tout comme La dama duende (1633-1635), l'une des plus drôles, et tant d'autres.

Pour distinguer l'énorme variété de sous-genres, les interprètes ajoutent à comedia diverses qualifications telles que "d'histoire", "sérieuse", "d'amour», "de saints", "burlesque», etc. Nos recherches récentes nous conduisent à nous intéresser à l'un de ces genres chez Calderón, celui de la comedia cómica. Ces pièces correspondent à ce qu'on appelle en français "comédie d'intrigue», où l'essentiel réside justement dans celle-ci: un coup de foudre qui provoque des malentendus, des déguisements, des ruses... des péripéties presque invraisemblables entre des personnages de classe moyenne-haute, jusqu'à la fin, usuellement heureuse. Notre étonnement partait de ce redoublement du comique dans leur désignation même. D’autant plus que, bien qu'ingénieuses, elles ne paraissent pas les plus drôles dans l'absolu - d'autres genres dits "brefs", tels entremeses et mojigangas, concentrent et produisent davantage le rire. Pourquoi alors emploie-t-on le comique au carré pour les nommer? Notre hypothèse est que "comédie» et «comique» valent ici pour "théâtre» et "théâtral ». L'affaire de la comédie comique - surtout celle de Calderón, ce maître de la métathéâtralité -, c'est au fond le théâtre: les personnages souvent se comportent en personnages de comédie, tout en explicitant ironiquement les conventions théâtrales. Dans

25. La Lettre à d'Alembert sur les spectacles (1758), où cette condamnation est décrétée, répond à l'article "Genève» de l'Encyclopédie, rédigé par d'Alembert où le philosophe regrettait l'absence d'une Comédie dans cette ville à tous autres égards louable. 
nombre de ces comédies, on peut lire entre les lignes une démonstration de ce que pour ces auteurs est le théâtre; dans leur langue: fingimiento, «feinte», «simulation». Lope de Vega l'avait d'ailleurs nettement établi dans son Arte nuevo de hacer comedias (1609): "L'objet de la tragédie est l'histoire, et celui de la comédie la feinte ${ }^{26}$. «Dramaturgie de la feinte» est la très pertinente formule de Marc Escola pour définir la matrice comique chez Corneille ${ }^{27}$. En effet, comme le remarque Guénoun, en français aussi le mot "comédie» vaut souvent pour le théâtre lui-même: "jouer la comédie», "Comédie-Française» ou encore la désignation des acteurs comme "comédiens» ${ }^{28}$. Par conséquent, lorsque les Espagnols désignent par "comédie» le théâtre profane en général, ils ne sont pas loin de cette idée: si le sujet de la comédie est, au fond, le théâtre, le propre du théâtre est, vice-versa, la comédie.

\section{Conclusion}

Tout en resserrant l'étendue de la philosophie et du théâtre, dans le contexte ancien de leur querelle, on est porté à les déterminer au moyen du concept de mimèsis et du versant comique du théâtre. Ce sont eux, leur visée critique et parodique, qui ont le plus poussé à notre sens vers l'idée de théâtre qu'on s'est faite par la suite. Du côté théorique, il suffit de considérer qu'on trouve le concept de mimèsis dans la définition aristotélicienne de tragédie et de comédie ${ }^{29}$. Nous avons tenté de mettre l'accent sur la composante comique originelle dans l'élaboration de ce concept, avant et chez Platon. Du côté pratique, en revanche, c'est la comédie qui apparait, lorsque le théâtre affronte la philosophie et se pense lui-même - se mettant en scène.

Pour conclure, cette réflexion peut être contractée dans le syllogisme suivant:

26. "Por argumento la tragedia tienel la historia, y la comedia el fingimiento;/ por eso fue llamada planipedia/ del argumento humilde, pues la hacía/ sin coturno y teatro el recitante./ Hubo comedias paliatas, mimos,/ togatas, atelanas, tabernarias,/ que también eran como agora varias». L. de Vega, Arte nuevo, vv. 111-118. Nous traduisons et soulignons.

27. M. Escola, «Présentation», p. 24.

28. D. Guénoun, "Théâtre et poésie. Propositions», p. 174.

29. Mimèsis praxeôs. Aristote, Poétique 6, $1449 \mathrm{~b} 24$. 
Si le théâtre est pensé comme mimèsis - comme le font Platon et d'Aristote -

et si la mimèsis est foncièrement comique - par son origine, sa pratique et sa nature -,

par le biais de la mimèsis, "la comédie est le devenir-théâtre du théâtre» ${ }^{30}$.

Quod erat demonstrandum.

\author{
María J. Ortega Máñez \\ Universität Wien
}

30. D. Guénoun, "Théâtre et poésie», p. 174. 


\section{BIBLIOGRAPHIE}

\section{Textes}

Aristophane, L'Assemblée des femmes, in Euvres complètes, V, texte éta-

bli par Victor Coulon, trad. Hilaire Van Daele, Paris, Les Belles Lettres, 1967.

-, Les Grenouilles, in Euvres complètes, IV, texte établi par Victor Coulon, trad. Hilaire Van Daele, Paris, Les Belles Lettres, 1967.

Aristote, La Poétique, trad. Roselyne Dupont-Roc, Jean Lallot, Paris, Seuil, 1980.

Platon, La République, trad. Pierre Pachet, Paris, Gallimard, 1993.

—, Le Politique, in Euvres complètes, sous la direction de Luc Brisson, Paris, Flammarion, 2008.

Vega de, Lope, Arte nuevo de hacer comedias este tiempo, ed. Felipe B. Pedraza Jiménez, Cuenca, Ediciones de la Universidad de Castilla-La Mancha, 2016.

\section{Travaux}

Chantraine, Pierre, Dictionnaire étymologique de la langue grecque, Paris, Klincksieck, 1999.

Deleuze, Gilles, Guattari, Félix, Qu'est-ce que la philosophie?, Paris, Éditions de Minuit, 1991.

Escola, Marc, "Présentation", in Corneille, La Place Royale, Paris, GF-Flammarion, 2019.

Ghiron-Bistagne, Paulette, Recherches sur les acteurs en Grèce antique, Paris, Les Belles Lettres, 1976.

Groneberg, Michael (éd.), Penser la scène. Études de lettres, 306 (2018/1).

—, «Introduction» in Michael Groneberg (éd.), Penser la scène. Études de lettres, 306 (2018/1), p. 7-14. 
—, «La mimesis: aspects ludiques et poétiques» in Michael Groneberg (éd.), Penser la scène. Études de lettres, 306 (2018/1), p. 145-167.

Guénoun, Denis, Le théâtre est-il nécessaire?, Paris, Circé, 1997.

—, "Théâtre et poésie. Propositions», in Michael Groneberg (éd.), Penser la scène. Études de lettres, 306 (2018/1), p. 170-179.

Halliwell, Stephen, The Aesthetics of Mimesis. Ancient Texts and Modern Problems, Princeton/Oxford, Princeton University Press, 2002.

Littré, Émile, Dictionnaire de la langue française, Chicago, Encyclopaedia Britannica Inc., 1994.

McCarey, Leo (réal.), Duck Soup, Paramount Pictures, 1933.

Palissot de Montenoy, Charles de, La comédie des Philosophes et autres textes, réunis, présentés et annotés par Olivier Ferret, SaintÉtienne, Publications de l'Université de Saint-Étienne, 2002.

Ricceur, Paul, Temps et récit, Paris, Seuil, 1983.

Sörвом, Göran, Mimesis and Art: Studies on the Origin and Early Development of an Aesthetic Vocabulary, Uppsala, Appelbergs, 1966. 